\title{
Lossy Metamaterials: No Effective Medium Properties without Noise
}

\author{
R.R.A.Syms ${ }^{1}$, O.Sydoruk ${ }^{1}$, L.Solymar ${ }^{1}$
}

\begin{abstract}
Lossy metamaterial elements act as sources of Johnson noise, making such materials inherently noisy. A coupled transmission line model capable of describing the effective medium properties, propagation and internal reflections, the internal noise distribution and the noise factor is developed. Two analyses are provided - numerical solution with limited physical insight and an approximation based on physical principles - and excellent agreement is obtained. It is shown that the internal noise spectrum is modified as it couples to the electromagnetic wave, and that there can be no change in permeability without an increase in the noise factor. This result implies that metamaterials will require careful evaluation of their noise performance before use in practical devices.
\end{abstract}

KEYWORDS: $\quad$ Metamaterial, Effective medium, Noise, Magneto-inductive wave

${ }^{1}$ EEE Dept., Imperial College London, Exhibition Road, London SW7 2AZ, UK

*Corresponding Author

TEL +44-207-594-6203 FAX +44-207-594-6308

Email r.syms@imperial.ac.uk 


\section{Introduction}

Metamaterials containing metallic resonant elements such as split-ring resonators (SRRs) and rods have received considerable attention, because of their ability to provide negative values of permittivity and permeability at frequencies up to the optical range, and the exciting potential applications arising therefrom [1-6]. The resonant elements are typically arranged in an array and a variety of effective medium theories [7-9] and homogenization methods [10-12] have been developed to recover the effective parameters with different unit cells. The simplest arrangement is a one-dimensional (1D) array [13-15]. In this case the interaction between an electromagnetic (EM) wave and the medium can be represented using a lumped-element model in which the EM wave is represented as an $\mathrm{L}-\mathrm{C}$ transmission line and the lossy resonant elements as $\mathrm{R}-\mathrm{L}-\mathrm{C}$ circuits $[16,17]$.

Considerable attention has been paid to conductor resistance, which introduces propagation loss, and to inter-element-coupling, which allows the propagation of lattice waves (for example, magneto-inductive [18] and electro-inductive [19] waves for magnetically and electrically coupled elements, respectively). However, all resistive elements must act as sources of Johnson noise [20-22], with a flat power spectral density. Dielectrics are an alternative source of loss and noise. However, a typical L-C resonator might comprise a inductor L with series resistance $\mathrm{R}$ and a lossy capacitor formed from a material with complex permittivity $\varepsilon=\varepsilon^{\prime}-\mathrm{j} \varepsilon^{\prime \prime}$. The latter may be represented as a lossless capacitor $C$ together with an equivalent series resistor $\varepsilon^{\prime \prime} /\left(\varepsilon^{\prime} \omega C\right)$, where $\omega=2 \pi f$ and $f$ is the frequency, which acts as a thermal noise source. Any associated noise will therefore have $1 /$ f spectral dependence and be most important at low frequency [23, 24]. 
Assuming a frequency-independent loss tangent, the contributions to resistance and noise from the two components will be equal when $\omega / \omega_{0}=\mathrm{Q}_{0} \varepsilon^{\prime \prime} / \varepsilon^{\prime}$, where $\omega_{0}=1 / \sqrt{ }(\mathrm{LC})$ is the angular resonant frequency and $\mathrm{Q}_{0}=\omega_{0} \mathrm{~L} / \mathrm{R}$ is the quality factor of the inductor. For typical values of $\mathrm{Q}_{0}$ $=100$ and $\varepsilon^{\prime \prime} / \varepsilon^{\prime}=10^{-4}, \omega / \omega_{0}=0.01$, implying that the inductor will be the dominant noise source near resonance. This conclusion will be invalid with purely dielectric resonators. However, dielectric noise may be treated in the analysis that follows by appropriately modifying the power spectral density of the noise sources.

The aspect of noise appears to have been largely ignored in metamaterials and may have a profound impact on any potential applications that involve signals. For example, a noisy electromagnetic wave incident on a metamaterial slab as shown in Figure 1a would be expected to exit the slab after suffering multiple reflection, attenuation and addition of further noise. While amplification may be used to overcome loss, it is considerably more difficult to mitigate the effects of noise.

It is well known that noise may also propagate as a wave in distributed electrical circuits [25-33]. Recently it was shown that the propagation of noise waves in magneto-inductive (MI) arrays alters the power spectral density of the noise quite dramatically [34], and similar effects have been noted in other types of electrical lattice [35]. However, interaction with an electromagnetic wave was omitted, and the effective medium properties were consequently ignored. Here, we extend the analysis of 1D magneto-inductive systems to include magnetic coupling to an EM wave in a magnetic metamaterial. We provide two models - a detailed numerical model and an analytic approximation - capable of simultaneously predicting the permeability and the noise 
performance of a medium with effective magnetic properties. In each case, multiple reflections are included. We show that the models agree in all their essential points, and that the effects are fundamentally linked. There can be no change in magnetic susceptibility caused by resonators formed from resistive elements without an increase in the noise factor, a conclusion that can be directly anticipated from the fluctuation-dissipation theorem [21, 36-38]. Here, we focus on the use of conductors to provide a negative value of $\mu_{\mathrm{r}}$. However similar conclusions are likely to be reached for negative index media that contain a second source of noise derived from the conductors providing a negative value of $\varepsilon_{\mathrm{r}}$.

In Section 2, we introduce the full model and a method of numerical solution for comparison with later approximations. In Section 3, we calculate the dispersion relations of the isolated and coupled systems. In Section 4, we use a perturbation solution to estimate the propagation constant of the electromagnetic wave and the effective permeability of the medium. In Section 5, we show how the power spectral density of the noise in the coupled resonator system may be found. In Section 6, we show how this noise is transferred to the EM wave and estimate the noise factor of a finite array. Conclusions are presented in Section 7. 


\section{2. $\quad$ Physical model}

Initial analysis of SRRs largely ignored their electric response [1]. However, it was subsequently shown that there could indeed be an electric response, depending on the internal arrangement of the SRRs and their orientation with respect to the electric field, and that this effect could be significant (see e.g. [39-41]). Such a response would of course give rise to a change in effective permittivity, and the inclusion of dielectric losses would allow this permittivity to be noisy. Further complications (such as electric coupling between the elements) allow magnetic coupling to the electromagnetic field to give rise to an electric response, and vice versa, and further possibilities for noise.

Here we emphasise that after a decade of active research and a very large number of papers exploring the wide range of possible effects in metamaterial lattices, the link between effective medium properties and noise has been ignored. As a result, although many exciting phenomena such as negative-index materials, epsilon-near-zero materials and transformation optics have been explored, and applications such as cloaking and electrically small devices have been proposed, there has been no attempt to investigate what may be a significant performance limitation.

In the first paper on the subject, there is a strong case to begin with the simplest possible analysis. Such an approach allows a relatively complicated calculation to be presented clearly, without being obscured by the details of multiple couplings. In many cases magnetic effects are dominant, and furthermore can be engineered to be so through careful design of the elements and the lattice $[39,42]$. We therefore restrict ourselves to magnetic coupling between the EM wave 
and the elements and between the elements themselves. With this assumption, the effect of the SRRs may be entirely attributed to changes in permeability.

The physical model assumed consists of an EM wave propagating in a waveguide past a onedimensional array of coplanar resonant elements such as SRRs. The EM wave is polarized so that its magnetic field may interact with the resonators, which then provide an effective magnetic medium. Figure 1b shows a low-frequency equivalent circuit, which consists of a pair of coupled lines [16]. The resonators are represented as a 1-D lattice of lumped-element circuits of period a, with inductance $\mathrm{L}$, capacitance $\mathrm{C}$ and resistance $\mathrm{R}$, coupled to nearest neighbours by mutual inductance M. Such a line supports MI waves. Due to the resistors, each element contains an independent Johnson noise source $\mathrm{V}_{\mathrm{NR}}$. The EM wave is represented by a lossless transmission line of the same period, with parameters $L^{\prime}=\mu_{0} \mathrm{a}$ and $\mathrm{C}^{\prime}=\varepsilon_{0} \mathrm{a}$, where $\mu_{0}$ and $\varepsilon_{0}$ are the permeability and permittivity of free space, which is terminated with a matched load. The EM wave is derived from a signal voltage source $V_{S}$ with output resistance $Z_{0}$, where $Z_{0}$ is the characteristic impedance of the line. Source noise is represented by the Johnson noise source $\mathrm{V}_{\mathrm{NS}}$, which is assumed to arise from the source impedance. All noise sources are assumed to have the same temperature. Coupling between the lines is represented by mutual inductance M', which will be negative here. Currents in the $\mathrm{n}^{\text {th }}$ element of the EM and MI lines are $\mathrm{J}_{\mathrm{n}}$ and $\mathrm{I}_{\mathrm{n}}$, respectively. For ease of comparison with standard Johnson noise expressions, all voltages and currents are specified by rms values.

A mathematical model corresponding to Figure 1b may be constructed by using Kirchhoff's voltage law to generate a set of equations relating currents to voltages. We assume $\mathrm{N}_{1}$ sections of 
EM transmission line, followed by $\mathrm{N}_{2}$ sections of interaction region and finally $\mathrm{N}_{3}$ sections of transmission line. However, $\mathrm{N}_{1}$ and $\mathrm{N}_{3}$ need only be sufficient to allow later extraction of parameters. The total number of sections is therefore $\mathrm{N}_{4}=\mathrm{N}_{1}+\mathrm{N}_{2}+\mathrm{N}_{3}$, and there are $\mathrm{N}_{5}=\mathrm{N}_{4}+$ $\mathrm{N}_{2}$ equations to solve. At the input of the EM line, we will have at angular frequency $\omega=2 \pi f$ :

$$
\left(\mathrm{Z}_{0}+\mathrm{j} \omega \mathrm{L}^{\prime}\right) \mathrm{J}_{1}+\left(\mathrm{J}_{1}-\mathrm{J}_{2}\right) / \mathrm{j} \omega \mathrm{C}^{\prime}=\mathrm{V}_{1}
$$

Here $\mathrm{V}_{1}$ is an input voltage, which might be due either to signal or to source noise. Between the input of the EM line and the interaction region $\left(1<\mathrm{n}<\mathrm{N}_{1}\right)$, and between the interaction region and the load $\left(\mathrm{N}_{1}+\mathrm{N}_{2}<\mathrm{n}<\mathrm{N}_{4}\right)$, we will have:

$$
j \omega L^{\prime} J_{n}+\left(J_{n}-J_{n+1}\right) / j \omega C^{\prime}-\left(J_{n-1}-J_{n}\right) / j \omega C^{\prime}=0
$$

Within the interaction region $\left(\mathrm{N}_{1}<\mathrm{n}<\mathrm{N}_{1}+\mathrm{N}_{2}\right)$ we will have

$$
\begin{gathered}
j \omega L^{\prime} J_{n}+\left(J_{n}-J_{n+1}\right) / j \omega C^{\prime}-\left(J_{n-1}-J_{n}\right) / j \omega C^{\prime}+j \omega M^{\prime} I_{n}=0 \\
(R+j \omega L+1 / j \omega C) I_{n}+j \omega M\left(I_{n+1}+I_{n-1}\right)+j \omega M^{\prime} J_{n}=V_{N R n}
\end{gathered}
$$

Here the voltages $\mathrm{V}_{\mathrm{NRn}}$ are due to Johnson noise in the MI waveguide elements. Finally, at the load, we will have:

$$
\mathrm{Z}_{0}^{*} \mathrm{~J}_{\mathrm{N} 3}-\left(\mathrm{J}_{\mathrm{N} 3-1}-\mathrm{J}_{\mathrm{N} 3}\right) / \mathrm{j} \omega \mathrm{C}^{\prime}=0
$$


Equations 1-4 can clearly be written in matrix form, as $\underline{V}=\underline{Z} \underline{I}$, where $\underline{Z}$ is an $N_{5} \times N_{5}$ matrix containing impedances and $\underline{\mathrm{V}}$ and $\underline{\mathrm{I}}$ are $\mathrm{N}_{5}$-element column vectors of voltages and currents, respectively. Their solutions can yield a number of results for comparison with later analytic approximations. For example, the effects of each voltage source may be found. Where these generate travelling waves, forward and backward waves may be separated to yield dispersion characteristics for each one, and reflection and transmission coefficients may also be found. In addition, they may be solved repetitively, to find the power dissipated in the load when either the voltage $\mathrm{V}_{1}$, or one of the voltages $\mathrm{V}_{\mathrm{NRn}}$ is present in isolation. The results can then be scaled, to represent either a signal voltage, or a voltage arising from Johnson noise. Once this has been done, addition of powers may be used to find the internal noise or the signal-to-noise ratio (SNR) at the output (which may then be compared with the SNR at the input to find the noise factor F). 


\section{Dispersion relations}

In general, the EM wave will encounter a finite line of resonators. However, we start by considering infinite systems. For the transmission line in isolation, and in the absence of any voltage sources, the assumption of travelling wave solutions to Equations 2 leads to the dispersion equation:

$$
1-2 \omega_{0}{ }^{\prime 2} / \omega^{2}+2\left(\omega_{0}, 2 / \omega^{2}\right) \cos \left(k_{\mathrm{EM}} \mathrm{a}\right)=0
$$

Here $\omega_{0}^{\prime}=1 / \sqrt{ }\left(\mathrm{L}^{\prime} \mathrm{C}^{\prime}\right)$ and $\mathrm{k}_{\mathrm{EM}}$ is the propagation constant of the EM wave. This $\omega-\mathrm{k}$ relation has the well-known sinusoidal variation, providing low-pass propagation up to a maximum angular frequency $2 \omega_{0}^{\prime}$. At low frequencies, it approximates to the straight-line relation $k_{\mathrm{EM}} \mathrm{a}=\omega / \omega_{0}{ }^{\prime}$, and leads to the real-valued characteristic impedance $Z_{0}=\sqrt{ }\left(L^{\prime} / C^{\prime}\right)=\sqrt{ }\left(\mu_{0} / \varepsilon_{0}\right)$.

Similarly, for a chain of resonators in isolation, this approach leads to the dispersion equation for MI waves [18]:

$$
1-\omega_{0}{ }^{2} / \omega^{2}-j \omega_{0} / \omega Q_{0}+\kappa \cos \left(k_{M I} a\right)=0
$$

Here $\omega_{0}=1 / \sqrt{ }(\mathrm{LC})$ is the angular resonant frequency of the elements, and $\mathrm{Q}_{0}=\omega_{0} \mathrm{~L} / \mathrm{R}$ is their quality factor, $\kappa=2 \mathrm{M} / \mathrm{L}$ is the coupling coefficient and $\mathrm{k}_{\mathrm{MI}}$ is the propagation constant of the MI wave. For negative $\kappa$, the waves are backward. In the absence of losses, this $\omega$-k relation provides band-pass propagation over the range $1 / \sqrt{ }(1+|\kappa|) \leq \omega / \omega_{0} \leq 1 /(1-|\kappa|)$. Propagation 
losses are low at mid-band, rising rapidly at the band edges. The effect of a finite Q-factor is to allow additional out-of-band propagation, albeit with very high loss.

For the two lines together, Equations 3 yield the dispersion relation [16]:

$$
\left\{1-2 \rho^{2} \omega_{0}^{2} / \omega^{2}+2 \rho^{2}\left(\omega_{0}^{2} / \omega^{2}\right) \cos (\mathrm{ka})\right\}\left\{1-\omega_{0}^{2} / \omega^{2}-\mathrm{j} \omega_{0} / \omega_{\mathrm{Q}_{0}}+\kappa \cos (\mathrm{ka})\right\}-\mathrm{q}^{2}=0
$$

Here $\mathrm{q}^{2}=\mathrm{M}^{\prime 2} / \mathrm{LL}$ is the normalised coupling coefficient between the EM and MI waves, and we have also introduced the ratio $\rho=\omega_{0}{ }^{1} / \omega_{0}$. The dispersion equation may be solved to yield a $\omega-\mathrm{k}$ diagram with two branches. The thin lines in Figure 2a show this characteristic for the lossless case, for the example parameters $\kappa=-0.2, \mathrm{q}^{2}=0.02$ and $\rho=20$. These results are in full agreement with previous theories, e.g. [7]. The thick lines show the corresponding results obtained for the two uncoupled systems, to which the coupled solutions are asymptotic. In this frequency range, the asymptotes are straight. For the coupled system, there is a gap between the branches near $\omega / \omega_{0}=1 / \sqrt{ }(1+\kappa)=1.118$; however, the introduction of loss allows propagation in this region. 


\section{4. $\quad$ Effective medium properties}

Since the effects of the resonators in this restricted model are entirely magnetic, we first note that the relative permeability can be found from the dispersion relation as $\mu_{\mathrm{r}}=\left(\mathrm{ka} / \mathrm{k}_{\mathrm{EM}} \mathrm{a}\right)^{2}$, where ka corresponds to the electromagnetic branch of the coupled system. In general, $\mathrm{k}$ is complex and can be written as $\mathrm{k}=\mathrm{k}^{\prime}-\mathrm{jk} \mathrm{k}^{\prime}$. Its frequency dependence may of course be found, by solving Equation 7. The data points in Figure $2 \mathrm{~b}$ show the variation of $\omega$ with $\mathrm{k}^{\prime}$ obtained for $\kappa=-0.2, \mathrm{Q}_{0}$ $=100, \mathrm{q}^{2}=0.02$ and $\rho=20$. The result is similar to Figure $2 \mathrm{a}$, but the addition of loss has now allowed propagation in the gap. Identical results can be obtained by numerical solution of the circuit equations for a finite array, separation of the currents into different waves and extraction of the relevant propagation constant.

The data points in Figure 3a show the corresponding frequency variation of the real and imaginary parts of $\mu_{\mathrm{r}}$. The variations show typical resonant behaviour, shifted in frequency by the magnetic coupling between the elements to a higher resonant frequency $\omega_{0} / \sqrt{ }(1+\kappa)$. Although MI wave propagation is supported over a wide band, the effects on the EM wave are restricted to the narrow frequency range where the two are almost synchronous. The real part of the relative permeability can clearly become negative in this region.

Although the numerical value of ka will be used in subsequent calculations, we now present a simple analytic approximation for comparison with published homogenization theories. To do so we assume that the resonators provide a loading that alters the propagation constant of the EM 
wave to $\mathrm{k}=\mathrm{k}_{\mathrm{EM}}+\Delta \mathrm{k}$. Substituting into (7), eliminating terms using (5), neglecting second order terms and using the low-frequency approximation for $\mathrm{k}_{\mathrm{EM}} \mathrm{a}$ we obtain:

$$
\mathrm{ka}=\omega / \rho \omega_{0}-\left(\mathrm{q}^{2} / 2 \rho\right)\left(\omega / \omega_{0}\right) /\left\{1+\kappa-\omega_{0}{ }^{2} / \omega^{2}-\mathrm{j} \omega_{0} / \omega \mathrm{Q}_{0}\right\}
$$

The full line in Figure $2 \mathrm{~b}$ shows the variation of $\omega$ with $\mathrm{k}^{\prime}$ found in this way, for the same parameters as before. There is clearly excellent agreement with the numerical solution. The agreement worsens as $\mathrm{Q}_{0}$ or $\mathrm{q}^{2}$ rises, since the effect on the EM wave is larger, but for moderate parameters the perturbation solution provides a very reasonable approximation.

Dividing (8) by $\mathrm{k}_{\mathrm{EM}} \mathrm{a}$ and squaring the result, we can find the relative permeability as:

$$
\mu_{\mathrm{r}}=\left[1-\left(\mathrm{q}^{2} / 2\right) /\left\{1+\kappa-\omega_{0}^{2} / \omega^{2}-\mathrm{j} \omega_{0} / \omega \mathrm{Q}_{0}\right\}\right]^{2}
$$

If the interaction term $\mathrm{q}^{2}$ is small enough, we then get:

$$
\mu_{\mathrm{r}}=1-\mathrm{q}^{2} /\left\{1+\kappa-\omega_{0}^{2} / \omega^{2}-\mathrm{j} \omega_{0} / \omega \mathrm{Q}_{0}\right\}
$$

Equation 10 is clearly identical to expressions commonly found in the literature, if $\mathrm{q}^{2}$ is identified with the so-called 'filling factor' (see $[1,7]$, or the discussion of five separate models for $\mu_{\mathrm{r}}$ in Sec. 2.8 of [43]). The full lines in Figure 3a show its predictions, for the same parameters as before. Once again there is excellent agreement with the numerical result (the data points). 
A change in $\mu_{\mathrm{r}}$ from unity will of course give rise to reflection at the array boundaries, as shown in Figure 3b. Since the model allows a pair of coupled modes, transmission and reflection are both in general accompanied by mode conversion: an electromagnetic wave incident on the array boundary will actually excite not only an E-M like transmitted wave, but also a MI-like wave. Here for simplicity we ignore the latter, and assume amplitude reflection and transmission coefficients $r_{1}, t_{1}, r_{2}$ and $t_{2}$ for the EM-like wave in standard forms valid for low frequency current waves incident on a discontinuity between transmission line sections with different magnetic properties:

$$
\begin{array}{ll}
\mathrm{r}_{1}=\left(\mathrm{k}_{\mathrm{EM}}-\mathrm{k}\right) /\left(\mathrm{k}_{\mathrm{EM}}+\mathrm{k}\right) & \mathrm{t}_{1}=2 \mathrm{k}_{\mathrm{EM}} /\left(\mathrm{k}_{\mathrm{EM}}+\mathrm{k}\right) \\
\mathrm{r}_{2}=\left(\mathrm{k}-\mathrm{k}_{\mathrm{EM}}\right) /\left(\mathrm{k}_{\mathrm{EM}}+\mathrm{k}\right) & \mathrm{t}_{2}=2 \mathrm{k} /\left(\mathrm{k}_{\mathrm{EM}}+\mathrm{k}\right)
\end{array}
$$

The lines in Figure $3 b$ show the frequency dependence of $\left|r_{1}\right|^{2}$ and $\left|t_{1}\right|^{2}$ for the same parameters as Figure 3a, which shows that these coefficients differ from zero and unity only when the relative permeability also differs significantly from unity. As before, the data points show results obtained from the numerical model. Good agreement is again obtained, with only slight discrepancies where the interaction is at its strongest. Furthermore, examination of the numerical model shows that the MI-like wave is indeed only weakly excited. 


\section{Internal noise}

We now consider the internal noise, which arises from Johnson noise in the resonators. If the coupling to the EM wave is weak, little of this noise will be transferred. We therefore begin by considering the resonator array in isolation, assuming this time that it is finite and extends from $\mathrm{n}$ $=1$ to $\mathrm{n}=\mathrm{m}$. For comparison with the full numerical model, we will take $\mathrm{N}_{2}=\mathrm{m}$.

At frequencies low enough for the noise spectrum to be flat, each of the internal noise sources will have rms voltage $\mathrm{V}_{\mathrm{NR}}$ such that $\mathrm{V}_{\mathrm{NR}} \mathrm{V}_{\mathrm{NR}} *=4 \mathrm{KTRdf}$ in a small bandwidth df [20, 21]. Here $\mathrm{K}$ is Boltzmann's constant and $\mathrm{T}$ is absolute temperature. Elsewhere [34], we have shown that each source in isolation will excite travelling waves, which are reflected at the ends of a finite array as standing noise waves as shown in Figure 4a. These waves may then be coupled to the electromagnetic line as shown in Figure 4b. However, we will postpone consideration of the more complicated geometry until the following section.

The driven response of the finite array can be found using a simple theory previously presented for a rather different problem (point excitation in near-field imaging devices [44]). This paper showed how the complete response to excitation of an arbitrary lossy magneto-inductive array could be found as an expansion in terms of the eigenmodes of the same array when lossless and undriven. For the particular case of a regular array with rectangular boundaries, the mode shapes and eigenvalues can be found analytically, allowing calculations to be performed extremely simply. For the 1-D array here, the current in the $\mathrm{r}^{\text {th }}$ element due to a source in the $\mathrm{s}^{\text {th }}$ element can be found as: 


$$
\mathrm{I}_{\mathrm{rs}}=\left(\mathrm{V}_{\mathrm{NR}} / \mathrm{R}\right)_{\mathrm{v}-1} \Sigma^{\mathrm{m}} \mathrm{i}_{\mathrm{sv}} \mathrm{i}_{\mathrm{rv}} / \lambda_{v}
$$

Here $i_{s v}$ is the normalised shape of the $v^{\text {th }}$ resonant mode of the equivalent lossless line, given by:

$$
\mathrm{i}_{\mathrm{su}}=\sqrt{ }\{2 /(\mathrm{m}+1)\} \sin \{\mathrm{s} v \pi /(\mathrm{m}+1)\} \text { with } v=1,2 \ldots \mathrm{m}
$$

The term $i_{r_{v}}$ is similar, but has s replaced with $\mathrm{r}$. The terms $\lambda_{v}$ are:

$$
\lambda_{v}=\left\{1+j Q_{0}\left(\omega / \omega_{0}\right)\left(\omega_{0}{ }^{2} / \omega_{v}^{2}-\omega_{0}{ }^{2} / \omega^{2}\right)\right\}
$$

Here $\omega_{v}$ is the angular resonant frequency of the $v^{\text {th }}$ eigenmode, given by:

$$
\omega_{v}{ }^{2} / \omega_{0}{ }^{2}=1 / \sqrt{ }\{1+\kappa \cos [v \pi /(m+1)]\}
$$

From Equation 12, we may obtain:

$$
\mathrm{I}_{\mathrm{rS}} \mathrm{I}_{\mathrm{rs}} *=\left.\left(\mathrm{V}_{\mathrm{NR}} \mathrm{V}_{\mathrm{NR}} * / \mathrm{R}^{2}\right)\right|_{\mathrm{v}-1} \Sigma^{\mathrm{m}} \mathrm{i}_{\mathrm{Sv}} \mathrm{i}_{\mathrm{rv}} /\left.\lambda_{\mathrm{v}}\right|^{2}
$$

Incoherently summing the effect of all noise sources then yields:

$$
\mathrm{I}_{\mathrm{r}} \mathrm{I}_{\mathrm{r}} * \mathrm{R}=\left.\left(\mathrm{V}_{\mathrm{NR}} \mathrm{V}_{\mathrm{NR}} * / \mathrm{R}\right)_{\mathrm{s}=1} \Sigma^{\mathrm{m}}\right|_{\mathrm{v}-1} \Sigma^{\mathrm{m}} \mathrm{i}_{\mathrm{sv}} \mathrm{i}_{\mathrm{rv}} /\left.\lambda_{\mathrm{v}}\right|^{2}
$$

Using the orthonormality of the modes, we then obtain:

$$
\mathrm{I}_{\mathrm{r}} \mathrm{I}_{\mathrm{r}} * \mathrm{R}=\left(\mathrm{V}_{\mathrm{NR}} \mathrm{V}_{\mathrm{NR}} * / \mathrm{R}\right)_{\mathrm{v}=1} \Sigma^{\mathrm{m}} \mathrm{i}_{\mathrm{rv}}{ }^{2} /\left|\lambda_{\mathrm{v}}\right|^{2}
$$

This result may be written as $\mathrm{I}_{\mathrm{r}} \mathrm{I}_{\mathrm{r}} * \mathrm{R}=\mathrm{P}\left(\mathrm{V}_{\mathrm{NR}} \mathrm{V}_{\mathrm{NR}} * / \mathrm{R}\right)$. Here $\mathrm{P}$ is a normalised power spectral density (PSD) describing a modification to the otherwise flat spectrum of Johnson noise caused by the reactive elements in the array, given by: 


$$
\mathrm{P}(\omega, \mathrm{r})={ }_{\mathrm{v}=1} \Sigma^{\mathrm{m}} \mathrm{i}_{\mathrm{rv}}{ }^{2} /\left|\lambda_{v}\right|^{2}
$$

This result may be written in full as:

$$
\mathrm{P}=\{2 /(\mathrm{m}+1)\}_{\mathrm{v}=1} \Sigma^{\mathrm{m}} \sin ^{2}\{\mathrm{rv} \pi /(\mathrm{m}+1)\} /\left\{1+\left[\mathrm{Q}_{0}\left(\omega / \omega_{0}\right)\left(\omega_{0}{ }^{2} / \omega_{v}{ }^{2}-\omega_{0}{ }^{2} / \omega^{2}\right)\right]^{2}\right\}
$$

The PSD was discussed extensively in [34], and shown to depend on the number of elements m, the position $r$, the coupling coefficient $\kappa$ and the Q-factor. Figure 5a shows its frequency variation for a 9-element array with $\kappa=-0.2$ and $\mathrm{Q}_{0}=100$. The thick and thin lines show results at the array edge $(r=9)$ and centre $(r=5)$ respectively. In each case, there is a set of resonances. At the edge, all nine modes are visible. However, only five can be seen at the centre, since the antisymmetric modes all have zeros at this point and hence make no contribution. Figure $5 \mathrm{~b}$ shows similar results, for a 49-element array. Now the resonances are starting to form a continuum. At the array centre, the PSD has assumed a characteristic shape, which tends to the homogeneous result obtained in an infinite array with peaks at the band edges where the propagation losses and modal density are both high. The same results are obtained using the numerical model, if the EM wave is omitted. 


\section{Noise factor}

We now consider how the internal noise is coupled to the EM wave. One obvious question is whether the internal noise of the array, which exists over the whole MI band, is transferred to the EM wave unaltered. We also estimate the noise factor F of the array, which is given by:

$$
\mathrm{F}=\left(\mathrm{S}_{\mathrm{In}} / \mathrm{N}_{\mathrm{In}}\right) /\left(\mathrm{S}_{\mathrm{Out}} / \mathrm{N}_{\text {Out }}\right)
$$

Here $\mathrm{S}_{\text {In }}$ and $\mathrm{N}_{\text {In }}$ and $\mathrm{S}_{\text {Out }}$ and $\mathrm{N}_{\text {Out }}$ are the signal and noise powers at the input and output. In a linear device, the noise factor is an intrinsic property and does not depend on the signal power. A key test of the performance of metamaterial devices will be how their attenuation and noise factor compare with those of more conventional solutions.

In addition to the full numerical model, we develop an analytic model to explain the physics. Because so many simultaneous equations are involved, construction of such a model is only possible if sweeping approximations are made. Effectively, these amount to assuming weak coupling between the EM wave and the array, so that the perturbations to the propagation constant of the former and the noise distribution of the latter are both relatively small.

Even an approximate model should include reflection at the array boundaries. For example, an incident EM wave should be transmitted at the array input, multiply reflected an infinite number of times, and then transmitted at the output. The amplitude $\mathrm{J}_{\mathrm{m}}$ of the current representing the wave at the output of the array may be related to the amplitude $\mathrm{J}_{0}$ at the input by the sum-of-allpaths method as: 


$$
\mathrm{J}_{\mathrm{m}}=\mathrm{J}_{0} \mathrm{t}_{1} \mathrm{t}_{2} \exp (-\mathrm{jmk} \mathrm{a})\left\{1+\mathrm{r}_{2}^{2} \exp (-2 \mathrm{jmka})+\mathrm{r}_{2}{ }^{4} \exp (-4 \mathrm{jmka}) \ldots\right\}
$$

Summing the exponential terms allows this result to be written as $\mathrm{J}_{\mathrm{m}}=\mathrm{J}_{0} \mathrm{t}$, where $\mathrm{t}$ is an overall amplitude transmission coefficient, given by:

$$
\mathrm{t}=\mathrm{t}_{1} \mathrm{t}_{2} \exp (-\mathrm{jmka}) /\left\{1-\mathrm{r}_{2}^{2} \exp (-2 \mathrm{jmka})\right\}
$$

Clearly Equation 23 describes a Fabry-Perot response that allows resonance whenever the roundtrip phase change in the array is a whole number of multiples of $2 \pi$. However, since the reflection coefficient $r_{2}$ is significantly different from unity only at frequencies when there is also loss, high-finesse resonance is unlikely to occur.

As a result of these effects, both the signal and the input noise power alter as they propagate through the array. Hence we can write:

$$
\begin{gathered}
\mathrm{S}_{\text {Out }}=\mathrm{S}_{\text {In }}|\mathrm{t}|^{2} \\
\mathrm{~N}_{\text {Out }}=\mathrm{N}_{\text {In }}|\mathrm{t}|^{2}+\mathrm{N}_{\text {Add }}
\end{gathered}
$$

Here $\mathrm{N}_{\text {In }}$ is the available noise power of the source and $\mathrm{N}_{\text {Add }}$ is the additive noise generated in the resonator array. The noise factor is therefore:

$$
\mathrm{F}=1+\mathrm{N}_{\text {Add }} /\left(\mathrm{N}_{\text {In }}|\mathrm{t}|^{2}\right)
$$


For a Johnson noise source at the input, we can immediately write $\mathrm{N}_{\mathrm{in}}=\mathrm{KTdf}$ for a small bandwidth df [22]. However, to find $\mathrm{N}_{\text {add }}$, we must consider how noise is transferred from the resonator array to the EM wave. For weak coupling, a single current $\mathrm{I}_{\mathrm{n}}$ in the $\mathrm{n}^{\text {th }}$ array element will excite a pair of waves into the EM line, travelling in either direction as shown in Figure 4b. It is simple to show that the amplitude of each wave is $\kappa_{\mathrm{v}} \mathrm{I}_{\mathrm{n}}$, where $\kappa_{\mathrm{v}}$ is a coupling coefficient given by:

$$
\kappa_{\mathrm{v}}=-(\mathrm{j} / \mathrm{ka})\left(\mathrm{M}^{\prime} / 2 \mathrm{~L}^{\prime}\right)\left(\omega / \rho \omega_{0}\right)^{2}
$$

Excitation of these waves is due to the currents $\mathrm{I}_{\mathrm{rs}}$ previously given in (12). If coupling of noise back into the array is neglected, the additive noise is the total power dissipated by all such waves in the load, taking care to account for any coherent effects and multiple reflections. For example, summing all the waves due to a single noise source in the $\mathrm{s}^{\text {th }}$ resonant element yields an EM current wave leaving the array with total amplitude:

$$
\mathrm{J}_{\mathrm{TS}}=\kappa_{\mathrm{r}=1} \Sigma^{\mathrm{m}} \mathrm{I}_{\mathrm{rs}}\left[\exp \{-\mathrm{j}(\mathrm{m}-\mathrm{r}) \mathrm{ka}\}+\mathrm{r}_{2} \exp \{-\mathrm{j}(\mathrm{m}+\mathrm{r}) \mathrm{ka}\}\right] \mathrm{t}_{\mathrm{N}}
$$

Here the first exponential describes contributions from all those noise waves that are initially forward going, the second describes all waves that are initially backward-going and $t_{\mathrm{N}}$ is an overall amplitude transmission coefficient that can again be found by the sum-of-all-paths method as: 


$$
\mathrm{t}_{\mathrm{N}}=\mathrm{t}_{2}\left\{1+\mathrm{r}_{2}^{2} \exp (-2 \mathrm{jmka})+\mathrm{r}_{2}^{4} \exp (-4 \mathrm{jmka}) \ldots\right\}
$$

Or as:

$$
\mathrm{t}_{\mathrm{N}}=\mathrm{t}_{2} /\left\{1-\mathrm{r}_{2}^{2} \exp (-2 \mathrm{jmka})\right\}
$$

Clearly, the transmission coefficients of the input wave and the additive noise wave are related, as $\mathrm{t}_{\mathrm{N}}=\left(\mathrm{t} / \mathrm{t}_{1}\right) \exp (+\mathrm{jmka})$ and $\left|\mathrm{t}_{\mathrm{N}}\right|^{2}=\left|\mathrm{t} / \mathrm{t}_{1}\right|^{2} \exp (2 \mathrm{mk}$ "a $)$. Consequently, Fabry-Perot resonance will affect both together, and many of the effects will cancel in the final noise factor.

Incoherent addition of the effects of all such noise sources then involves a sum of the form $\mathrm{J}_{\mathrm{T}} \mathrm{J}_{\mathrm{T}} *$ $={ }_{\mathrm{s}=1} \Sigma^{\mathrm{m}} \mathrm{J}_{\mathrm{Ts}} \mathrm{J}_{\mathrm{Ts}} *$. Combination with the results of the previous section shows that this can be written as:

$$
\mathrm{J}_{\mathrm{T}} \mathrm{J}_{\mathrm{T}} *=\mathrm{p}\left(\kappa_{\mathrm{N}} \kappa_{\mathrm{N}} * 4 \mathrm{KTdf} / \mathrm{R}\right)\left|\mathrm{t}_{\mathrm{N}}\right|^{2}
$$

Here the function $\mathrm{p}$ is given by:

$$
\mathrm{p}(\omega, \mathrm{m})=\left.\left.{ }_{\mathrm{s}=1} \Sigma^{\mathrm{m}}\right|_{\mathrm{r}=1} \Sigma^{\mathrm{m}}{ }_{\mathrm{v}=1} \Sigma^{\mathrm{m}}\left(\mathrm{i}_{\mathrm{sv}} \mathrm{i}_{\mathrm{rv}} / \lambda_{\mathrm{v}}\right)\left[\exp \{-\mathrm{j}(\mathrm{m}-\mathrm{r}) \mathrm{ka}\}+\mathrm{r}_{2} \exp \{-\mathrm{j}(\mathrm{m}+\mathrm{r}) \mathrm{ka}\}\right]\right|^{2}
$$

The additive noise power dissipated in the load may then be found as

$$
\mathrm{N}_{\mathrm{Add}}=\mathrm{Z}_{0} \mathrm{~J}_{\mathrm{T}} \mathrm{J}_{\mathrm{T}} *
$$


Substitution of (32) into (25) shows that the noise factor must be:

$$
\mathrm{F}=1+\mathrm{p}\left(4 \kappa_{\mathrm{N}} \kappa_{\mathrm{s}} * \mathrm{Z}_{0} / \mathrm{R}\right) \exp \left(2 \mathrm{mk} \mathrm{k}^{\prime \prime}\right) /\left|\mathrm{t}_{1}\right|^{2}
$$

Minor substitutions then allow this result to be written in the following form:

$$
\mathrm{F}=1+\mathrm{p}\left(\mathrm{q}^{2} /|\mathrm{ka}|^{2}\right)\left(\mathrm{Q}_{0} \omega / \omega_{0}\right)\left(\omega / \rho \omega_{0}\right)^{3} \exp (2 \mathrm{mk} \mathrm{k}) /\left|\mathrm{t}_{1}\right|^{2}
$$

The full line in Figure 6a shows the frequency variation of the noise factor obtained using this model, for a 9-element array with the same parameters as before $\left(\kappa=-0.2, \mathrm{Q}_{0}=100, \mathrm{q}^{2}=0.02\right.$ and $\rho=20$ ). F is close to unity, except at peaks near a discrete set of frequencies that correspond to noise resonances in the magneto-inductive array. Comparison with Figure 5a shows that only odd-order modes can be distinguished, with successively decreasing amplitude. Consequently, the power spectral density of the noise is altered very significantly as it is transferred to the EM wave. The full line in Figure $6 \mathrm{~b}$ shows corresponding results for a 49-element array. Now only a single peak may be seen. This peak corresponds to coupling at the frequency at which the effective permeability is most different from unity in Figure 3a.

The explanation for this behaviour can be found in the form of the function p. For large arrays, the second exponential in (31) may effectively be disregarded, since it will be heavily reduced by attenuation. The summation then represents a phased addition of a single set of terms, and only those adding coherently (which requires the EM and MI waves to be synchronous) will give a significant result. This condition coincides with the condition required for a magnetic effect. Unfortunately, it also corresponds to a frequency range in which the internal noise density is high 
at the centre of the array. Furthermore, both $\mu_{\mathrm{r}}-1$ (the magnetic susceptibility) and $\mathrm{F}-1$ (the excess noise factor) are proportional to $\mathrm{q}^{2}$, showing that the two are inescapably linked.

The data points in Figures $6 \mathrm{a}$ and $6 \mathrm{~b}$ show the corresponding results from the numerical model. Although there are some small discrepancies in the peak heights, the qualitative agreement is excellent. This result is striking considering the sweeping approximations made in deriving the analytic model, and confirm its essential validity. Effectively it can provide the same results as a combination of multiple solutions of large numbers of simultaneous equations, and therefore represents a homogenization procedure. For large arrays, the noise factor is determined almost entirely by the propagation constant $\mathrm{k}$ and the power transmission coefficient $\left|\mathrm{t}_{1}\right|^{2}$. Here we have used the value of k, so any discrepancies are largely due to the errors in the transmission coefficient previously shown in Figure 3b. For large m, the analytic model provides a slight overestimate of $\mathrm{F}$; however, both models predict an exponential increase of $\mathrm{F}$ - 1 with distance as would be expected.

The peak value of $\mathrm{F}$ in Figure $6 \mathrm{a}$ is approximately 2 , corresponding to a noise figure of $\approx 3 \mathrm{~dB}$. Clearly, this result is obtained at the peak of the absorption band, an undesirable operating point. However, the results are clearly considerably worse - and completely unrealistic for device applications - in Figure 6b. Better noise figures are clearly obtained off-resonance, but in this frequency range the effect of the medium on the wave is reduced. Further calculations show that the peak attenuation and noise factor increase with $\mathrm{Q}_{0}$. However, the frequency range over which both are large reduces at the same time, increasing the range of useable performance. 
As presented, the expression for the noise factor is relatively clumsy, and further simplifications will certainly be possible. Several terms are slowly varying with frequency, and others tend to simple limits as $\mathrm{m}$ rises. However, since thick slabs have high loss, careful thought will have to be given as to how usable loss and noise performance may be combined with negative parameters.

For a single Johnson noise source, the available noise power (i.e. the power that can be transferred to a matched load) is $\mathrm{P}_{\mathrm{av}}=\mathrm{KTB}$, where $\mathrm{B}$ is the bandwidth [22]. At room temperature $(\mathrm{T}=293 \mathrm{~K})$, we therefore obtain $\mathrm{P}_{\mathrm{av}}=4 \times 10^{-14} \mathrm{~W}, 4 \times 10^{-13} \mathrm{~W}$ and $4 \times 10^{-12} \mathrm{~W}$ for example bandwidths of $\mathrm{B}=10 \mathrm{MHz}, 100 \mathrm{MHz}$ and $1 \mathrm{GHz}$, respectively. These powers are clearly small, and the cumulative effect of a many noise sources would be required to obtain a large total noise power. However, it should be emphasised that in any noise factor calculation one is effectively making comparisons between a relatively small additive noise and a source noise power of similar magnitude. 


\section{Conclusions}

We have demonstrated a physical model capable of simultaneously describing the effective magnetic properties and noise of a one-dimensional array of magnetically coupled resonators containing resistive elements. The model shows that any coupling between a resonator array and an electromagnetic wave that gives rise to an alteration in effective permeability also transfers noise to the wave, with a spectral distribution that is related to the power spectral density of the Johnson noise in the array. The transferred noise is concentrated in exactly the frequency range where significant changes in effective permeability occur, so that all such media must be inherently noisy.

We have found a full solution of the model relying on generalized Kirchhoff equations. Since it is only a numerical solution leading to no physical insight, we have also solved the problem of noise transfer by a set of approximations that render the mathematical problem tractable and offer a clear physical picture. The full solution has been compared with the analytic approximations for the propagation constant, relative permeability, Fresnel coefficients, internal noise distribution and noise factor, and excellent agreement has been obtained in each case.

The model was chosen to be as simple as possible, subject to the condition that it should yield a realistic description of noise. We have restricted the model to purely magnetic interaction between the EM wave and the metamaterial array, and to purely magnetic interaction between the elements. Future generalisations might involve modification of the equivalent circuit to include modelling of:

i) Conducting rods (to model noise in negative index materials) 
ii) Electrical interaction between the EM wave and the elements

iii) Electrical interaction between the elements

iv) Non-nearest neighbour interactions between the elements

v) $\quad 2 \mathrm{D}$ and $3 \mathrm{D}$ arrays

vi) Loss and noise associated with lossy dielectric elements

Calculations could again be performed directly using a numerical approach, or analytically in terms of noise waves. In each case, care would be required to extract the full range of anisotropic effective medium properties, but many techniques exist for doing so. However, we emphasise that the effects of any additional noise sources are likely to be cumulative, since these are uncorrelated.

Even without these developments, we may draw conclusions about the use of gain. Gain may certainly be used to compensate for attenuation, but only front-end amplification will lead to much improvement in the noise factor because distributed amplification will also amplify any noise due to the medium itself, and, even worse will create additional noise due to the amplification process. The implications are that all devices designed to exploit negative index media that contain lossy elements should be critically examined for their noise performance. 


\section{8. $\quad$ References}

1. Pendry J.B., Holden A.J., Robbins D.J., Stewart W.J. "Magnetism from conductors and enhanced nonlinear phenomena" IEEE Trans. Micr. Theory Tech. 47, 2075-2084 (1999)

2. Smith D.R., Padilla W.J., Vier D.C., Nemat-Nasser S.C., Schultz S. "Composite medium with simultaneously negative permeability and permittivity” Phys. Rev. Lett. $\underline{84}$, 4184-4187 (2000)

3. Gokkavas M., Guven K., Bulu I., Aydin K., Penciu R.S., Kafesaki M., Soukoulis C.M., Ozbay E. "Experimental demonstration of left-handed metamaterial operating at $100 \mathrm{GHz}$ " Phys. Rev. B 73, 193103 (2006)

4. Zhou J., Koschny Th., Kafesaki M., Economou E.N., Pendry J.B., Soukoulis C.M. "Saturation of the magnetic response of split-ring resonators at optical frequencies" Phys. Rev. Lett. 95, 223902 (2005)

5. Shalaev V.M., Cai W., Chettiar U.K., Yuan H.-K., Sarychev A.K., Drachaev V.P., Kildishev A.V. "Negative index of refraction in optical metamaterials" Optics Letts. 30 , 3356-3358 $(2005)$

6. Dolling G., Wegener M., Soukoulis C.M., Linden S. "Negative-index metamaterial at 780-nm wavelength” Optics Letts. $\underline{32}$, 53-55 (2007)

7. Smith D.R., Vier D.C., Kroll N., Schultz S. "Direct calculation of permeability and permittivity for a left-handed metamaterial” Appl. Phys. Lett. 77, 2246-2248 (2000)

8. Koschny T., Kafesaki M., Economou E.N., Soukoulis C.M. "Effective medium theory of lefthanded metamaterials” Phys. Rev. Lett. 93, 107402-1-4 (2004)

9. Chen H., Ran L., Huangfu J. "Equivalent circuit model for left-handed metamaterials" J. Appl. Phys. 100, 024915 (2006) 
10. Smith D.R., Pendry J.B. "Homogenization of metamaterials by field averaging” JOSA B $\underline{23}$, $391-403(2006)$

11. Silveirinha M.G. "Metamaterial homogenization approach with application to the characterization of microstructured composites with negative parameters” Phys. Rev. B $\underline{75}$, $115104(2007)$

12. Baena J.D., Jelinek L., Marques R., Silveirinha M. "Unified homogenization theory for magneto-inductive and electroinductive waves in split-ring metamaterials” Phys. Rev. A $\underline{78},-$ $12842(2008)$

13. Marques R., Martel J., Mesa F., Medina F. "Left-handed-media simulation and transmission of EM waves in subwavelength split-ring-resonator-loaded metallic waveguides" Phys. Rev. Lett. $\underline{89}, 183901-1-4(2002)$

14. Martin F., Bonache J., Falcone F., Sorolla M., Marques R. "Split ring resonator-based lefthanded coplanar waveguide” Appl. Phys. Lett. 83, 4652-4654 (2003)

15. Belov P.A., Simovski C.R. "Subwavelength metallic waveguides loaded by uniaxial resonant scatterers" Phys. Rev. E 72, 036618 (2005)

16. Syms R.R.A., Shamonina E., Kalinin V., Solymar L. "A theory of metamaterials based on periodically loaded transmission lines: interaction between magneto-inductive and electromagnetic waves" J. Appl. Phys. 97, 064909 (2005)

17. Baena J.D., Bonache J., Martin F., Marques R.S., Falcone F., Lopetegi T., Laso M.A.G., Garcia-Garcia J., Gil I., Portillo M.F., Sorolla M. “Equivalent-circuit models for split-ring resonators coupled to planar transmission lines" IEEE Trans. Micr. Theory Tech. $\underline{53}, 1451$ $1461(2005)$

18. Shamonina E., Kalinin V.A., Ringhofer K.H., Solymar L. "Magneto-inductive waveguide" Elect. Lett. 38 , 371-373 (2002) 
19. Beruete M., Falcone F., Freire M.J., Marques R., Baena J.D. "Electroinductive waves in chains of complementary metamaterials” Appl. Phys. Lett. $\underline{88}, 083503$ (2006)

20. Johnson J.B. “Thermal agitation in electrical conductors" Phys. Rev. 32, 87-109 (1928)

21. Nyquist H. "Thermal agitation of electric charge in conductors" Phys. Rev. 32, 110-113 (1928)

22. Pierce J.R. "Physical sources of noise" Proc. IRE $\underline{44}, 601-608$ (1956)

23. Israeloff N.E., Wang X. "High-sensitivity dielectric polarization noise measurements" Rev. Sci. Inst. $\underline{68}, 1543-1546(1997)$

24. Kleinpenning T.G.M. "Relation between $1 / \mathrm{f}$ noise and frequency-dependent loss tangent" J. Phys. Condens. Matt. 10, 4245-4256 (1998)

25. Bauer H., Rothe H. "Der aquivalente rauschvierpol als wellenvierpol” Arch. Elekt. Übertragung 10, 241-252 (1956)

26. Rothe H., Dahlke W. “Theory of noisy fourpoles” Proc. IRE 44, 811-818 (1956)

27. Penfield P. "Wave representation of amplifier noise" IRE Trans. Circuit Theory CT-9, 83-84 (1962)

28. Bosma H. "On the theory of linear noisy systems" PhD Thesis, Technische Hogeschool Eindhoven (1967)

29. Meys R.P. "A wave approach to the noise properties of linear microwave devices" IEEE Trans. Micr. Theory Tech. 26, 34-37 (1976)

30. Hecken R.P. “Analysis of linear noisy two-ports using scattering waves" IEEE Trans. Micr. Theory Tech. MTT-29, 997-1004 (1981)

31. Kanaglekar N.G., McIntosh R.E., Bryant W.E. "Wave analysis of noise in interconnected multiport networks" IEEE Trans. Micr. Theory Tech. MTT-35, 112-116 (1987) 
32. Withington S. "Scattered noise waves in microwave and mm-wave networks" Microwave J. $\underline{32}, 169-178(1989)$

33. Wedge S.W. "Noise waves and passive linear multiports" IEEE Micr. Guided Wave Letts. $\underline{1}$, $117-119(1991)$

34. Syms R.R.A., Solymar L. "Noise in metamaterials" J. Appl. Phys. 109, 124909 (2011)

35. Syms R.R.A., Solymar L. "Noise waves in periodic electrical lattices" Proc. $5^{\text {th }}$ Int. Cong. on Advanced Electromagnetic Materials in Microwaves and Optics, Barcelona, Spain, Oct. 1015 , pp. $27-29(2011)$

36. Callen H.B., Welton T.A. "Irreversibility and generalized noise” Phys. Rev. 83, 34-40 (1951)

37. Weber J. "Fluctuation dissipation theorem" Phys. Rev. 101, 1620-1626 (1956)

38. Kubo "The fluctuation dissipation theorem" Rep. Prog. Phys. 29, 255-284 (1966)

39. Marques R., Medina F., Rafii-El-Idrissi R. "Role of bianisotropy in negative permeability and left-handed metamaterials" Phys. Rev. B 65, 144440 (2002)

40. Marques R., Mesa F., Martel J., Medina F. "Comparative analysis of edge- and broadsidecoupled split ring resonators for metamaterials design - theory and experiments" IEEE Trans. Antennas Propag. 51, 2572-2581 (2003)

41. Smith D.R., Gollub J., Mock J.J., Padilla W.L., Schurig D. "Calculation and measurement of bianisotropy in a split ring resonator material” J. Appl. Phys. 100, 024507 (2006)

42. Baena J.D., Jelinek L., Marques R., Zehentner J. "Electrically small isotropic threedimensional magnetic resonators for metamaterial design” Appl. Phys. Lett. $\underline{88}, 134108$ (2006)

43. Solymar L., Shamonina E. "Waves in metamaterials" Oxford University Press, Oxford (2009) 44. Syms R.R.A., Shamonina E., Solymar L. "Near field image transfer by magneto-inductive arrays: a modal perspective" Metamaterials $\underline{5}, 8-25$ (2011) 


\section{8. $\quad$ Figures}

1. a) Accumulation of loss and noise in a RF metamaterial slab; b) coupled circuit model of an electromagnetic wave interacting magnetically with a chain of lossy resonators.

2. a) Dispersion characteristic of lossless coupled system, for $\kappa=-0.2, q^{2}=0.02$ and $\rho=20$ (thin lines); corresponding result for uncoupled EM and MI systems (thick lines). b) Dispersion characteristic of the electromagnetic wave in a lossy coupled system, for the parameters above and $\mathrm{Q}_{0}=100$. Points: numerical solution; line: approximate solution.

3. Frequency variation of a) the real and imaginary parts of the relative permeability and b) the transmission and reflection coefficients at the array input, for $\kappa=-0.2, \mathrm{Q}_{0}=100, \mathrm{q}^{2}=0.02$ and $\rho=20$. Points: numerical solution; lines: approximate solution.

4. Model for a) excitation of noise resonances in a resonator array, and b) coupling of the array noise to the EM wave.

5. Frequency variation of the normalised noise PSD, for a) a 9-element and b) a 49-element resonator array in isolation. In each case $\kappa=-0.2$ and $\mathrm{Q}_{0}=100$, and the thick lines show results for the array edge and the thin lines for the array centre.

6. Frequency variation of noise factor, for an electromagnetic wave coupled to a) a 9-element and b) a 49-element resonator array. In each case, $\kappa=-0.2, \mathrm{Q}_{0}=100, \mathrm{q}^{2}=0.02$ and $\rho=20$. Points: numerical solution, lines: approximate solution. 


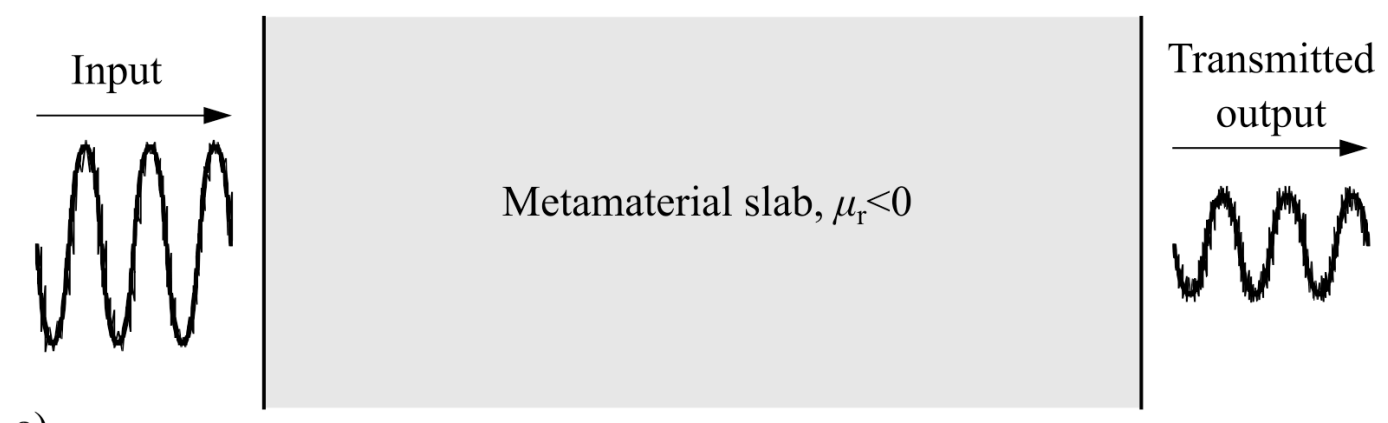

a)

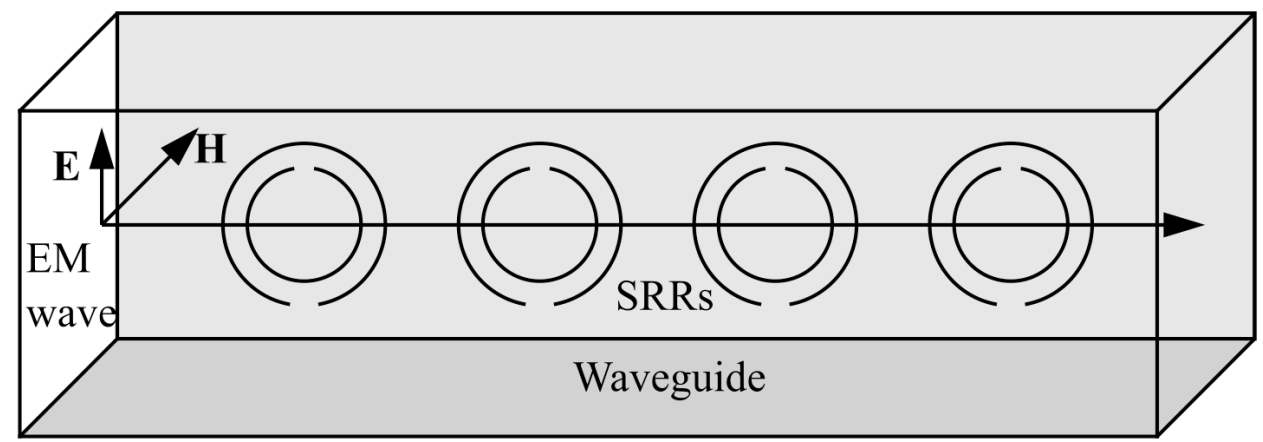

b)

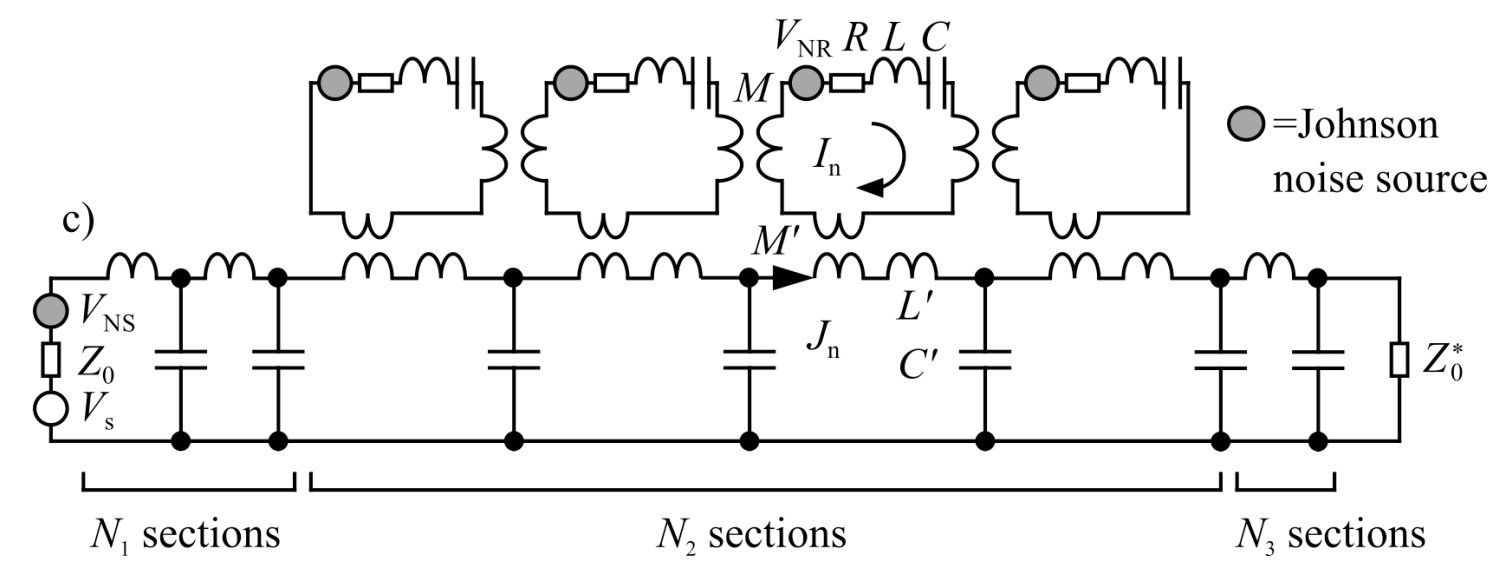

Figure 1. 

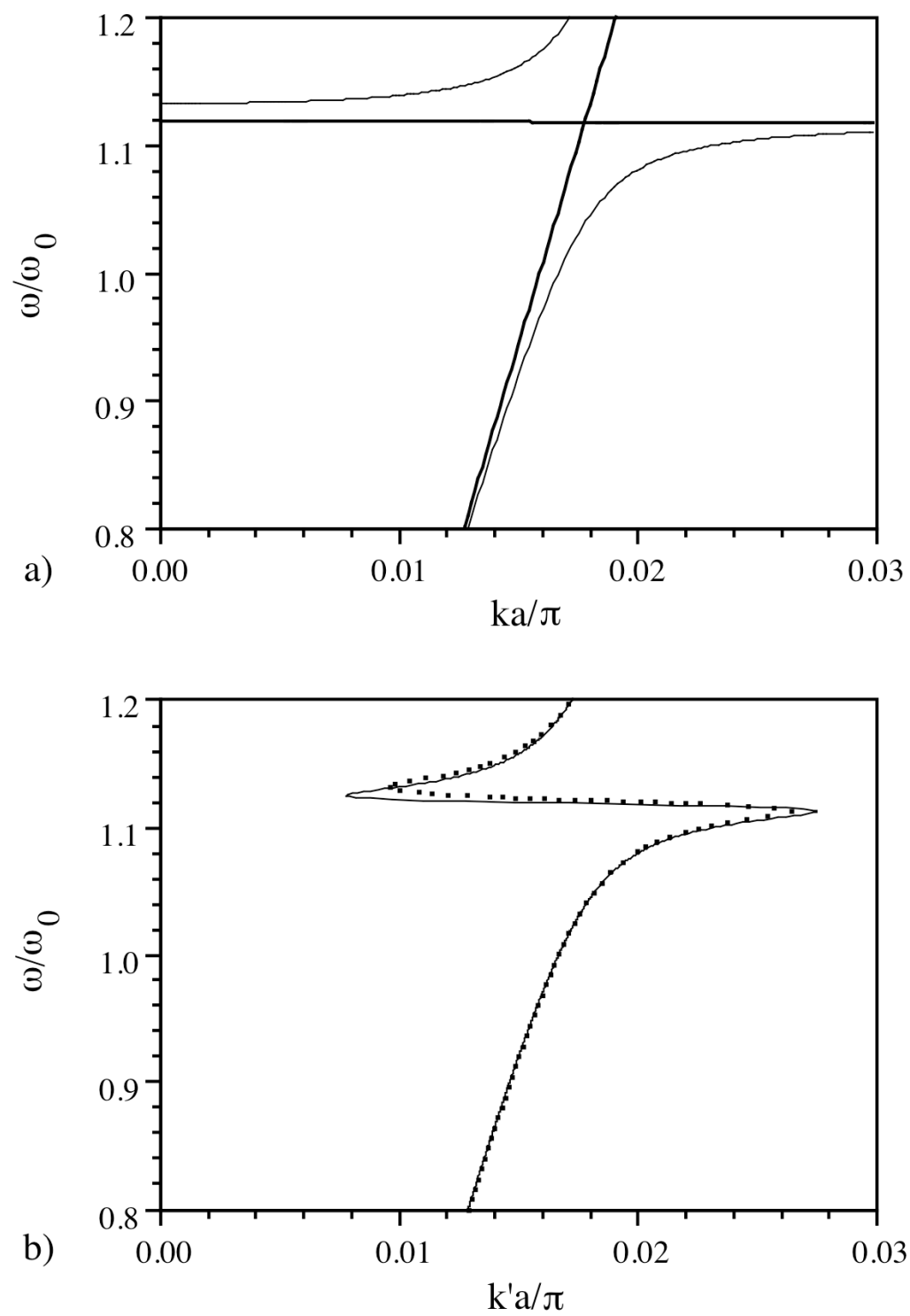

Figure 2. 

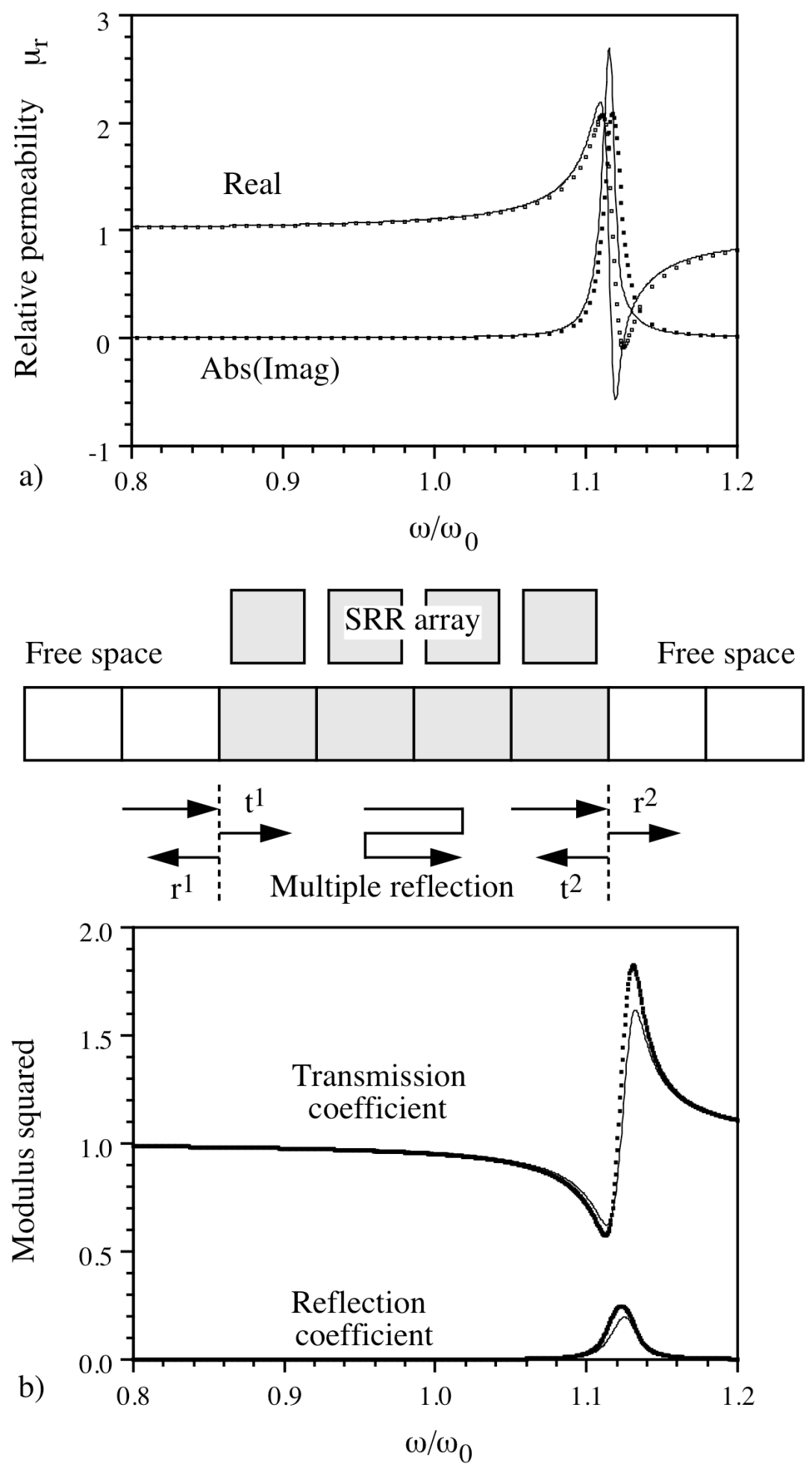

Figure 3. 


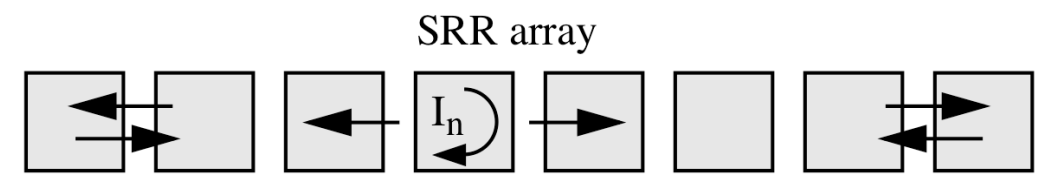

a)

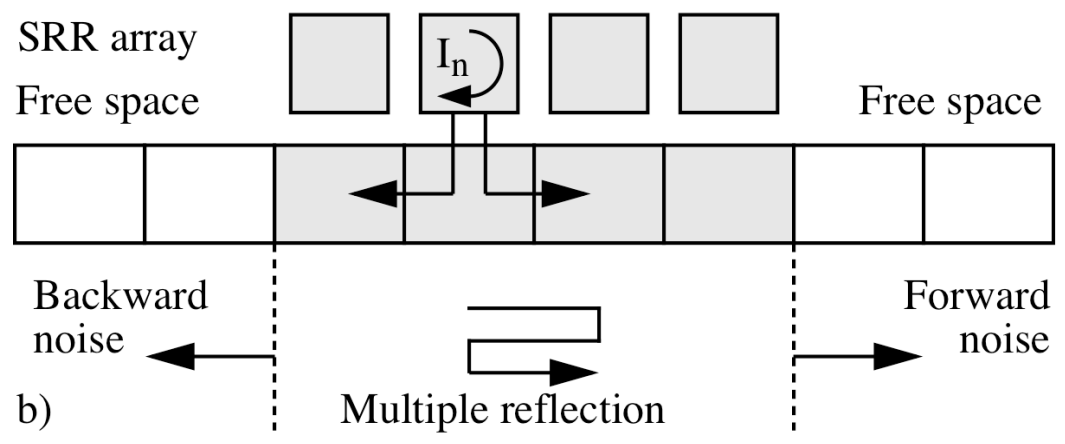

Figure 4. 

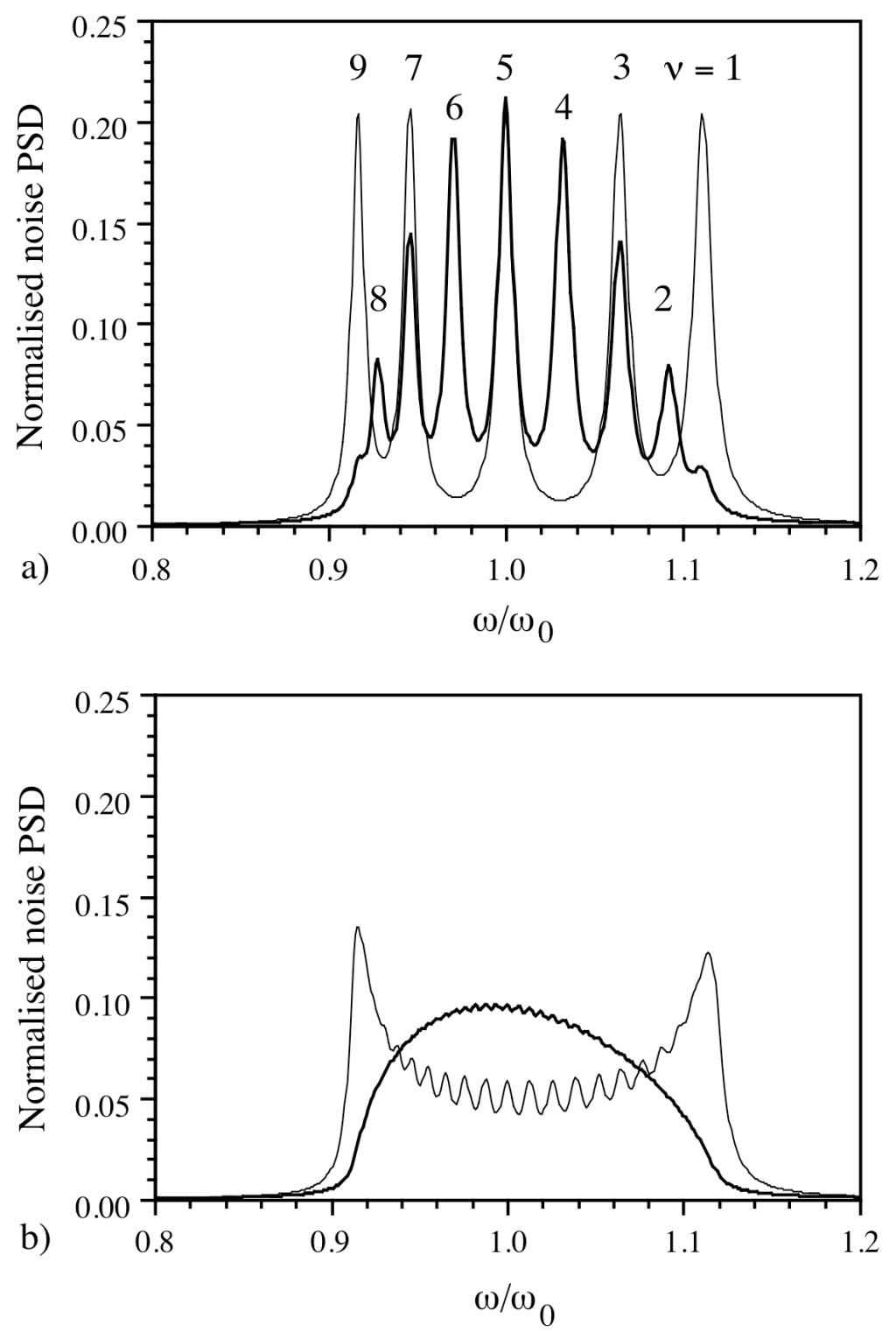

Figure 5. 

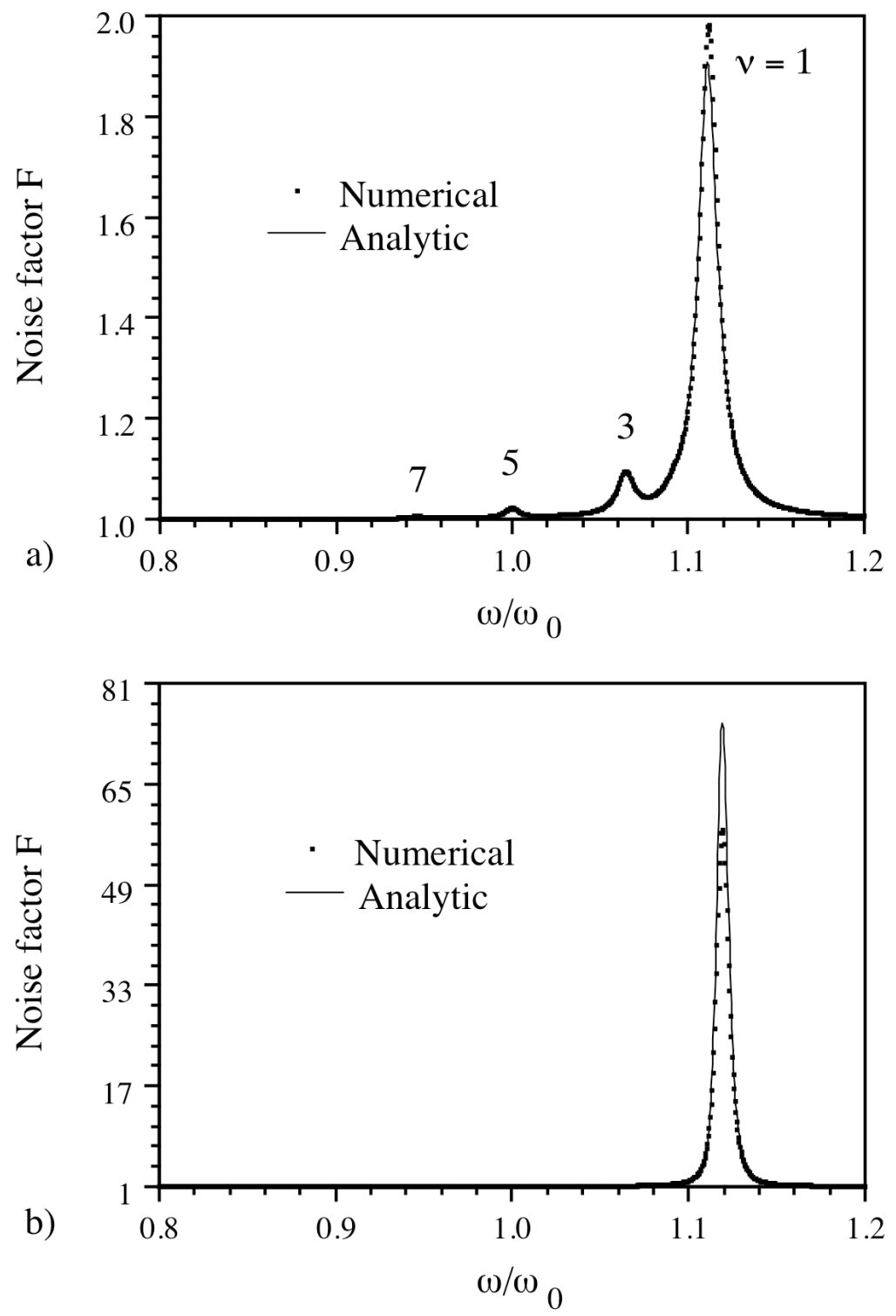

Figure 6. 